\title{
Characterizations of Marshall-Olkin Discrete Reduced Modified Weibull Distribution
}

\section{Gholamhossein G. Hamedani}

Department of Mathematics, Statistics and Computer Science, Marquette University, Milwaukee, USA

\section{Email address:}

g.hamedani@mu.edu

\section{To cite this article:}

Gholamhossein G. Hamedani. Characterizations of Marshall-Olkin Discrete Reduced Modified Weibull Distribution. International Journal of Statistical Distributions and Applications. Vol. 5, No. 1, 2019, pp. 1-4. doi: 10.11648/j.ijsd.20190501.11

Received: August 29, 2018; Accepted: April 22, 2019; Published: May 20, 2019

\begin{abstract}
Characterizing a distribution is an important problem in applied sciences, where an investigator is vitally interested to know if their model follows the right distribution. To this end, the investigator relies on conditions under which their model would follow specifically chosen distribution. Certain characterizations of the Marshall-Olkin discrete reduced modified Weibull distribution are presented to complete, in some way, their work.
\end{abstract}

Keywords: Discrete Marshall-Olkin distribution, Discrete Weibull Distribution, Discrete Distributions, Hazard Function, Characterizations

\section{Introduction}

The problem of characterizing a distribution is an important problem in applied sciences, where an investigator is vitally interested to know if their model follows the right distribution. To this end, the investigator relies on conditions under which their model would follow specifically chosen distribution. [1] introduced a new discrete probability model called "Marshall Olkin Discrete Reduced Modified Weibull (MDRMW)" distribution to compete against some of the well-known discrete distributions. In this very short note, we present two characterizations of MDRMW distribution based on: (i) conditional expectation of certain function of the random variable and (ii) the hazard rate function. We would like to mention here some of the recently introduced discrete distributions for the interested readers: (a) Discrete Logistic (DLOG) distribution [2]; (b) a discrete version of normal (DN1) distribution [3]; (c) Discrete Rayleigh (DR) distribution [4]; (d) Discrete Normal (DN2) [5]; (e) Discrete Weibull Type III (DWTIII) distribution [6]; (f) Discrete Gamma (DG) distribution [7]; (g) Discrete Beta-Exponential (DBE) distribution [8]; (h) Generalization of Geometric (GG) distribution [9]; (i) Discrete Generalization of Half-Normal (DGHN) distribution [10]; (j) Exponentiated Discrete Weibull (EDW) distribution [11]. The next four distributions were listed on page 4188 [12]: (k) Discrete Pareto (DP); (l) Discrete Haight's zeta (DHZ); (m) Discrete Half-Logistic
(DHL); (n) Discrete Truncated-Logistic (DTL); (o) Discrete Laplace (Double Exponential) (DL (DDE)) distribution [13]; (p) Discrete Geometric Weibull (DGW) distribution [14]; (q) Discrete Modified Weibull Extension (DMWE) distribution [15]. The next two distributions appear in [16]: (r) Discrete Modified Weibull Type I (DMWTI); (s) Discrete Modified Weibull Type II (DMWTII); (t) Discrete Reduced Modified Weibull (DRMW) distribution [17]; (u) Discrete Burr (DB) distribution [18]; (v) Discrete Inverse Rayleigh (DIR) [19]; (w) Another Discrete Burr (ADB) [20]; (x) Discrete Gumbel (DG); (y) Generalizations of Geometric (GOG) distributions [21] and (z) A New Generalized Poisson-Lindley (ANGPL) Distribution of [22]. For a detailed treatment of each one of these distributions and their domain of applicability, we refer the interested reader to the corresponding paper cited in the references. We certainly hope that the contents of this work will be useful to a good number of researchers whose model follows the MDRMW distribution. The cumulative distribution function (cdf), $F(x)$, and the corresponding probability mass function (pmf), $f(x)$, hazard rate function, $h_{F}(x)$, of MDRMW are given, respectively, by

$$
F(x ; \beta, q, b, c)=\frac{1-q^{(1+x)^{1 / 2}\left(1+b c^{x+1}\right)}}{1-\bar{\beta} q^{(1+x)^{1 / 2}\left(1+b c^{x+1}\right)}}, \quad x \in \mathbb{N}^{*}
$$




$$
\begin{aligned}
& f(x ; \beta, q, b, c)=\beta\left\{\frac{q^{x^{1 / 2}\left(1+b c^{x}\right)}}{1-\bar{\beta} q^{x^{1 / 2}\left(1+b c^{x}\right)}}-\frac{q^{(x+1)^{1 / 2}\left(1+b c^{x+1}\right)}}{1-\bar{\beta} q^{(x+1)^{1 / 2}\left(1+b c^{x+1}\right)}}\right\}, x \in \mathbb{N}^{*} \\
& h_{F}(x ; \beta, q, b, c)=\frac{q^{x^{1 / 2}\left(1+b c^{x}\right)}}{q^{(x+1)^{1 / 2}\left(1+b c^{x+1}\right)}} \times \frac{1-\bar{\beta} q^{(x+1)^{1 / 2}\left(1+b c^{x+1}\right)}}{1-\bar{\beta} q^{x^{1 / 2}\left(1+b c^{x}\right)}}-1, \quad x \in \mathbb{N}^{*}
\end{aligned}
$$

Where $\beta, b, c$ all positive and $q \in(0,1)$ are parameters and $\mathbb{N}^{*}=\{0\} \cup \mathbb{N}$ ( $\mathbb{N}$ is the set of all positive integers).

\section{Characterization Results}

We present our characterizations (i) and (ii) mentioned in the Introduction via two subsections 2.1 and 2.2, as follows.

\subsection{Characterizations of MDRMW in Terms of the Conditional Expectation of Certain Function of the Random Variable}

Proposition 2.1.1. Let $X: \Omega \rightarrow N^{*}$ be a random variable. The pmf of $X$ is (2) if and only if

$$
E\left\{\left(\frac{q^{X^{1 / 2}\left(1+b c^{X}\right)}}{1-\bar{\beta} q^{X^{1 / 2}\left(1+b c^{X}\right)}}+\frac{q^{(X+1)^{1 / 2}\left(1+b c^{X+1}\right)}}{1-\bar{\beta} q^{(X+1)^{1 / 2}\left(1+b c^{X+1}\right)}}\right) \mid X>k\right\}=\frac{q^{(k+1)^{1 / 2}\left(1+b c^{k+1}\right)}}{1-\bar{\beta} q^{(k+1)^{1 / 2}\left(1+b c^{k+1}\right)}}, \quad k \in \mathbb{N}^{*}
$$

Proof. If has pmf (2), then the left-hand side of (4) will be

$$
(1-F(k))^{-1} \sum_{x=k+1}^{\infty}\left[\beta\left\{\left(\frac{q^{x^{1 / 2}\left(1+b c^{x}\right)}}{1-\bar{\beta} q^{x^{1 / 2}\left(1+b c^{k}\right)}}\right)^{2}-\left(\frac{q^{(x+1)^{1 / 2}\left(1+b c^{x+1}\right)}}{1-\bar{\beta} q^{(x+1)^{1 / 2}\left(1+b c^{k+1}\right)}}\right)^{2}\right]=\left(\frac{1-\bar{\beta} q^{(k+1)^{1 / 2}\left(1+b c^{k+1}\right)}}{q^{(k+1)^{1 / 2}\left(1+b c^{k+1}\right)}}\right)\left(\frac{q^{(k+1)^{1 / 2}\left(1+b c^{k+1}\right)}}{1-\bar{\beta} q^{(k+1)^{1 / 2}\left(1+b c^{k+1}\right)}}\right)^{2}=\frac{q^{(k+1)^{1 / 2}\left(1+b c^{k+1}\right)}}{1-\bar{\beta} q^{(k+1)^{1 / 2}\left(1+b c^{k+1}\right)}}\right.
$$

Conversely, if (4) holds, then

$$
\sum_{x=k+1}^{\infty}\left\{\left(\frac{q^{x^{1 / 2}\left(1+b c^{x}\right)}}{1-\bar{\beta} q^{x^{1 / 2}\left(1+b c^{x}\right)}}+\frac{q^{(x+1)^{1 / 2}\left(1+b c^{k+1}\right)}}{1-\bar{\beta} q^{(x+1)^{1 / 2}\left(1+b c^{c+1}\right)}}\right) f(x)\right\}=(1-F(k))\left(\frac{q^{(k+1)^{1 / 2}\left(1+b c^{k+1}\right)}}{1-\bar{\beta} q^{(k+1)^{1 / 2}\left(1+b c^{k+1}\right)}}\right)=[1-F(k+1)+f(k+1)]\left(\frac{q^{(k+1)^{1 / 2}\left(1+b c^{k+1}\right)}}{1-\bar{\beta} q^{(k+1)^{1 / 2}\left(1+b c^{k+1}\right)}}\right)
$$

From (4), we also have

$$
\sum_{x=k+2}^{\infty}\left\{\left(\frac{q^{x^{1 / 2}\left(1+b c^{x}\right)}}{1-\bar{\beta} q^{x^{1 / 2}\left(1+b c^{x}\right)}}+\frac{q^{(x+1)^{1 / 2}\left(1+b c^{c+1}\right)}}{1-\bar{\beta} q^{(x+1)^{1 / 2}\left(1+b c^{x+1}\right)}}\right) f(x)\right\}=[1-F(k+1)]\left(\frac{q^{(k+2)^{1 / 2}\left(1+b c^{k+2}\right)}}{1-\bar{\beta} q^{(k+2)^{1 / 2}\left(1+b c^{k+2}\right)}}\right)
$$

Now, subtracting (6) from (5), we arrive at

$$
\begin{aligned}
& {[1-F(k+1)]\left\{\left(\frac{q^{(k+1)^{1 / 2}\left(1+b c^{k+1}\right)}}{1-\bar{\beta} q^{(k+1)^{1 / 2}\left(1+b c^{k+1}\right)}}\right)-\left(\frac{q^{(k+2)^{1 / 2}\left(1+b c^{k+2}\right)}}{1-\bar{\beta} q^{(k+2)^{1 / 2}\left(1+b c^{k+2}\right)}}\right)\right\}} \\
& =\left\{\begin{array}{l}
\left(\frac{q^{(k+1)^{1 / 2}\left(1+b c^{k+1}\right)}}{1-\bar{\beta} q^{(k+1)^{1 / 2}\left(1+b c^{k+1}\right)}}\right)+\left(\frac{q^{(k+2)^{1 / 2}\left(1+b c^{k+2}\right)}}{1-\bar{\beta} q^{(k+2)^{1 / 2}\left(1+b c^{k+2}\right)}}\right) \\
-\left(\frac{q^{(k+1)^{1 / 2}\left(1+b c^{k+1}\right)}}{1-\bar{\beta} q^{(k+1)^{1 / 2}\left(1+b c^{k+1}\right)}}\right)
\end{array}\right\} f(k+1)=\left(\frac{q^{(k+2)^{1 / 2}\left(1+b c^{k+2}\right)}}{1-\bar{\beta} q^{(k+2)^{1 / 2}\left(1+b c^{k+2}\right)}}\right) f(k+1) .
\end{aligned}
$$

From the last equality, we have 


$$
h_{F}(k+1)=\frac{f(k+1)}{1-F(k+1)}=\left\{\left(\frac{q^{(k+1)^{1 / 2}\left(1+b c^{k+1}\right)}}{1-\bar{\beta} q^{(k+2)^{1 / 2}\left(1+b c^{k+2}\right)}}\right) \times\left(\frac{1-\bar{\beta} q^{(k+2)^{1 / 2}\left(1+b c^{k+2}\right)}}{1-\bar{\beta} q^{(k+1)^{1 / 2}\left(1+b c^{k+1}\right)}}\right)\right\}-1, \quad x \in \mathbb{N}^{*}
$$

Which, in view of (3), implies that has pmf (2).

\subsection{Characterization of MDRMW Based on the Hazard Function}

Proposition 2.2.1. Let $X: \Omega \rightarrow N^{*}$ be a random variable. The pmf of $\quad$ is (2) if and only if its hazard rate function satisfies the difference equation

$$
h_{F}(k+1)-h_{F}(k)=\frac{q^{(k+1)^{1 / 2}\left(1+b c^{k+1}\right)}}{q^{(k+2)^{1 / 2}\left(1+b c^{k+2}\right)}} \times \frac{1-\bar{\beta} q^{(k+2)^{1 / 2}\left(1+b c^{k+2}\right)}}{1-\bar{\beta} q^{(k+1)^{1 / 2}\left(1+b c^{k+1}\right)}}-\frac{q^{k^{1 / 2}\left(1+b c^{k}\right)}}{q^{(k+1)^{1 / 2}\left(1+b c^{k+1}\right)}} \times \frac{1-\bar{\beta} q^{(k+1)^{1 / 2}\left(1+b c^{k+1}\right)}}{1-\bar{\beta} q^{k^{1 / 2}\left(1+b c^{k}\right)}}
$$

With the boundary condition $h_{F}(0)=\frac{1-q^{1+b c}}{\beta q^{1+b c}}$.

Proof. If has pmf (2), then clearly (7) holds. Now, if (7) holds, then for every $x \in N$, we have

$$
\begin{gathered}
\left.\sum_{k=0}^{x-1}\left[h_{F}(k+1)-h_{F}(k)\right]=\sum_{k=0}^{x-1} \begin{array}{l}
\frac{q^{(k+1)^{1 / 2}\left(1+b c^{k+1}\right)}}{q^{(k+2)^{1 / 2}\left(1+b c^{x+2}\right)}} \times \frac{1-\bar{\beta} q^{(k+2)^{1 / 2}\left(1+b c^{k+2}\right)}}{1-\bar{\beta} q^{(k+1) / 2\left(1+b c^{k+1}\right)}} \\
-\frac{q^{k^{1 / 2}\left(1+b c^{k}\right)}}{q^{(k+1)^{1 / 2}\left(1+b c^{k+1}\right)}} \times \frac{1-\bar{\beta} q^{(k+1)^{1 / 2}\left(1+b c^{k+1}\right)}}{1-\bar{\beta} q^{k^{1 / 2}\left(1+b c^{k}\right)}}
\end{array}\right\} \\
h_{F}(k+1)-h_{F}(0)=\frac{q^{x^{1 / 2}\left(1+b c^{x}\right)}}{q^{(x+1)^{1 / 2}\left(1+b c^{x+1}\right)}} \times \frac{1-\bar{\beta} q^{(x+1)^{1 / 2}\left(1+b c^{x+1}\right)}}{1-\bar{\beta} q^{x^{1 / 2}\left(1+b c^{x}\right)}}-\frac{1-\bar{\beta} q^{(1+b c)}}{\beta}=\frac{q^{x^{1 / 2}\left(1+b c^{x}\right)}}{q^{(x+1)^{1 / 2}\left(1+b c^{x+1}\right)}} \times \frac{1-\bar{\beta} q^{(x+1)^{1 / 2}\left(1+b c^{x+1}\right)}}{1-\bar{\beta} q^{1 / 2\left(1+b c^{x}\right)}}-1-\frac{1-q^{(1+b c)}}{\beta q^{(1+b c)}}
\end{gathered}
$$

In view of the fact that $h_{F}(0)=\frac{1-q^{1+b c}}{\beta q^{1+b c}}$, from the last equation we have

$$
h_{F}(x)=\frac{q^{x^{1 / 2}\left(1+b c^{x}\right)}}{q^{(x+1)^{1 / 2}\left(1+b c^{x+1}\right)}} \times \frac{1-\bar{\beta} q^{(x+1)^{1 / 2}\left(1+b c^{x+1}\right)}}{1-\bar{\beta} q^{x^{1 / 2}\left(1+b c^{x}\right)}}-1
$$

Which, in view of (3), implies that has pmf (2).

\section{Concluding Remark}

The problem of characterizing a distribution is an important one which can help the researcher to find out if their selected distribution is in fact the right one. This short note is intended to provide the characterizations of MDRMW distribution to complete, in some way, the work of Oloko et al. [1].

\section{References}

[1] Oloko, A. L., Asiribi, O. E., Dawoud, G. A., Omeike, M. O., Ajadi, N. A. and Ajayi, A. O., A new discrete family of reduced modified Weibull distribution, International J. of Statistical Distributions and Applications, 3, (2017), 25-31.

[2] Chakraborty, S. and Chakravarty, D., A new discrete probability distribution with integer support on $(-\infty, \infty)$, Commun. Statist.-Theory and Methods, (2015). To appear.

[3] Dasgupta, R., Cauchy equation on discrete domain and some characterization theorems, Theoret. Prob. Appl. 38, (1993), $520-524$.

[4] Roy, D., Discrete Rayleigh distribution, IEEE Transactions on Reliability, 53, (2004), 255-260.

[5] Roy, D., Discrete Rayleigh distribution, Commun. Statist. Theory-Methods, 32, (2003), 1871-1883.

[6] Padgett, W. J. and Spurrier, J. D., On discrete failure models, IEEE Transaction on Reliability, 34, (1985), 253-256.

[7] Abouammoh, A. M. and Alhazzani, N. S., On Discrete gamma distribution, Commun. Statist. Theory-Methods, 44, (2015), 3087-3098.

[8] Nekoukhou, V., Alamatsaz, M. H., Bidram, H. and Aghajani, A.H., Discrete beta-exponential distribution, Commun. Statist. Theory-Methods, 44, (2015), 2079-2091. 
[9] Gómez-Déniz, E., Another generalization of the geometric distribution, Test, 19, (2010), 399-415.

[10] Gómez-Déniz, E., Vázquez-Polo, F.J. and Gar' cia-Gar' cia, V., A discrete version of the half-normal distribution and its generalization with application, Stat Papers, 55, (2014), 497511.

[11] Nekoukhou, V. and Bidram, The exponentiated discrete Weibull distribution, SORT, 39, (2015), 127-146.

[12] Nair, N. U. and Sankaran, P. G., Odds function and odds rate for discrete lifetime distributions, Commun. Statist. Theory-Methods, 44, (2015), 4185 - 4202.

[13] Inusah, S. and Kozzubowski, T. J., A discrete analogue of the Laplace distribution, J. Stat. Planning. Inference, 136, (2006), 1090-1102.

[14] Bracquemond, C. and Gaudoin, O., A survey on discrete life time distributions, Int. J. Reliabil. Qual. Saf. Eng. 10, (2003), 69-98.

[15] Rezaei, R. A. H., Mohtashami, B. G. R. and Khorashiadzadeh, M., Some aspects of discrete telescopic hazard rate function in telescopic families, Econ. Qual. Control. 24, (2009), 35- 42.

[16] Chakraborty, S., Generating discrete analogues of continuous probability distributions-A survey of methods and constructions, J. of Statistical Distributions and Applications, DOI: 10.1186/s40488-015-0028-6 (2015).

[17] Almalki, S. J., Statistical analysis of lifetime data using new modified Weibull distributions, a thesis submitted to the University of Manchester for the degree of Doctor of Philosophy in the faculty of Engineering and Physical Sciences (2014).

[18] Khorashiadzadeh, M., Rezaei, R. A. H. and Mohtashami, B. G. R., Characterizations of life distributions using log-odds rate in discrete aging, Commun. Statist. Theory-Methods, 42, (2012), 76- 87.

[19] Hussain, T. and Ahmad, M., Discrete inverse Rayleigh distribution, Pak. J. Statist. 30, (2014), 203-222.

[20] Al-Huniti, A. A. and Al-Dayian, G. R., Discrete Burr type III distribution, Am. J. Math. Stat. 2, (2012), 145-152.

[21] Chakraborty, S. and Chakravarty, D., A discrete Gumbel distribution, arXiv: 1410.7568 [math.ST], 28 Oct. (2014).

[22] Bhati, D., Sastry, D. V. and Qadri, P. Z. M., A new generalized Poisson-Lindley distribution: applications and properties, Austrian J. of Statistics, 44, (2015), 35-51. 